\title{
The Quasi-Optimal Radial Basis Function Collocation Method: A Technical Note
}

\author{
Juan Zhang $\mathbb{D}^{1,2}$ Mei Sun $\mathbb{D}^{1,},{ }^{1,2}$ Enran Hou $\mathbb{D}^{1},{ }^{1}$ and Zhaoxing Ma $\mathbb{D}^{3}$ \\ ${ }^{1}$ School of Computer Science and Technology, Huaibei Normal University, Huaibei 235000, China \\ ${ }^{2}$ School of Information and Control Engineering, China University of Mining and Technology, Xuzhou 221116, China \\ ${ }^{3}$ School of Information and Control Engineering, Qingdao University of Technology, Qingdao 273400, China
}

Correspondence should be addressed to Enran Hou; houenran@163.com

Received 27 November 2020; Revised 22 December 2020; Accepted 4 February 2021; Published 25 September 2021

Academic Editor: Efthymios G. Tsionas

Copyright ( 92021 Juan Zhang et al. This is an open access article distributed under the Creative Commons Attribution License, which permits unrestricted use, distribution, and reproduction in any medium, provided the original work is properly cited.

The traditional radial basis function parameter controls the flatness of these functions and influences the precision and stability of approximation solution. The coupled radial basis function, which is based on the infinitely smooth radial basis functions and the conical spline, achieves an accurate and stable numerical solution, while the shape parameter values are almost independent. In this paper, we give a quasi-optimal conical spline which can improve the numerical results. Besides, we consider the collocation points in the Chebyshev-type which improves solution accuracy of the method with no additional computational cost.

\section{Introduction}

The multiquadric (MQ) radial basis function (RBF) collocation method, which is also named as Kansa's method [1], is a promising efficient numerical method for solving various problems governed by partial differential equations. As is known to all, the selection of shape parameter in the traditional MQ-RBF is very important in dealing with partial differential equations, and its small variation may cause severe differences to solutions [2]. The selection of optimal shape parameter in the RBF methods has long been a challenging task [3-7]. Several attempts and considerable progress have been made in literatures [8-11], but this question still remains a bottleneck for the MQ method application to practical problems.

Coupled RBFs (CRBFs) [12] are constructed by the multiquadric (MQ) and inverse multiquadric (IMQ) accompanied with the conical spline. For further investigations, a quasi-optimal conical spline is straightforwardly used for CRBF with the collocation points under the Chebyshev-type scheme. The novelty of the Chebyshev-type scheme lies in that the computational cost remains the same as traditional way with more accurate solutions, and there is no need to consider the fictitious points used in the other methods [13-16].

Section 2 gives the basic theory of the CRBF accompanied with a quasi-optimal conical spline and the collocation points under the Chebyshev-type scheme. Followed by Section 3, numerical results for two benchmark examples are used to show that the solution accuracy is improved as well as stability.

\section{The CRBF Method}

For brevity, we consider the boundary value problems for elliptic partial differential equation of second order:

$$
\begin{aligned}
& a(P) \frac{\partial^{2} u}{\partial x^{2}}+b(P) \frac{\partial^{2} u}{\partial y^{2}}+c(P) \frac{\partial u}{\partial x}+d(P) \frac{\partial u}{\partial y}+e(P) u=f(P) \\
& P=(x, y) \in \Omega \\
& u(P)=\bar{u}(P), P=(x, y) \in \Gamma_{D}
\end{aligned}
$$




$$
\frac{\partial u(P)}{\partial n}=\bar{q}(P), P=(x, y) \in \Gamma_{N},
$$

where $a(P), b(P), c(P), d(P)$, and $e(P)$ are prescribed coefficients, $\Omega \subset R^{2}$ is a two-dimensional physical domain, $\bar{u}(P)$ is the known boundary data on the Dirichlet boundary $\Gamma_{D}$, and $\bar{q}(P)$ is the known boundary data on the Neumann boundary $\Gamma_{N}$, with $\Gamma_{D} \cup \Gamma_{N}=\partial \Omega$ and $\Gamma_{D} \cap \Gamma_{N}=\phi$.

The basic theory of the CRBF method lies in that the numerical solution of the boundary value problem equations (1)-(3) can be given in the following general formulation:

$$
\widetilde{u}(P)=\sum_{j=1}^{M} \alpha_{j} \varphi\left(\left\|P-P_{j}\right\|_{2}\right)+r^{5},
$$

where $M$ is the total number of source points $\left\{P_{j}\right\}_{j=1}^{M}$ on the whole physical domain $\bar{\Omega}=\Omega \cup \partial \Omega,\left\{\alpha_{j}\right\}_{j=1}^{M}$ are the required coefficients, $\varphi\left(\left\|P-P_{j}\right\|_{2}\right)=\sqrt{r^{2}+c^{2}}$ is the MQ, and $r^{5}$ is the conical spline with $r_{j}=\left\|P-P_{j}\right\|$ the Euclidean norm distance between points $P=(x, y)$ and $P_{j}=\left(x_{j}, y_{j}\right)$. Here, different conical splines $\left(r^{7}, r^{9}, r^{11}\right)$ in equation (4) are considered for comparison, and the following numerical results will show that $r^{9}$ is the optimal choice, rather than $r^{5}$, with more accurate results as well as stability.

We denote $\left\{P_{j}\right\}_{j=1}^{M_{I}}$ the collocation points inside the domain $\Omega,\left\{P_{j}\right\}_{j=1}^{M_{D}}$ the collocation points on the Dirichlet boundary $\Gamma_{D}$, and $\left\{P_{j}\right\}_{j=1}^{M_{N}}$ the collocation points on the Neumann boundary $\Gamma_{N}$ with the total collocation number $M=M_{I}+M_{D}+M_{N}$.

Here, we consider the collocation points in the Chebyshev-type scheme, which is generated in the interval $(-1,1)$, instead of the traditional uniformly distributed source points. The novelty of the idea lies in that the computational cost remains the same as traditional way with more accurate solutions and there is no need to consider the fictitious points used in the other methods [13-16]. The definite generation of collocation points on each direction of the physical domain by Chebyshev-type is shown as follows:

$$
P_{j}=\cos \frac{\pi j}{n+1}, \quad 0 \leq j \leq n .
$$

For two-dimensional points, the configuration is shown in Figure 1.

For arbitrary interval $[a, b]$, an affine transformation can be used:

$$
P_{j}=\frac{1}{2}(a+b)+\frac{1}{2}(b-a) \cos \frac{j \pi}{n+1}, \quad 1 \leq j \leq n .
$$

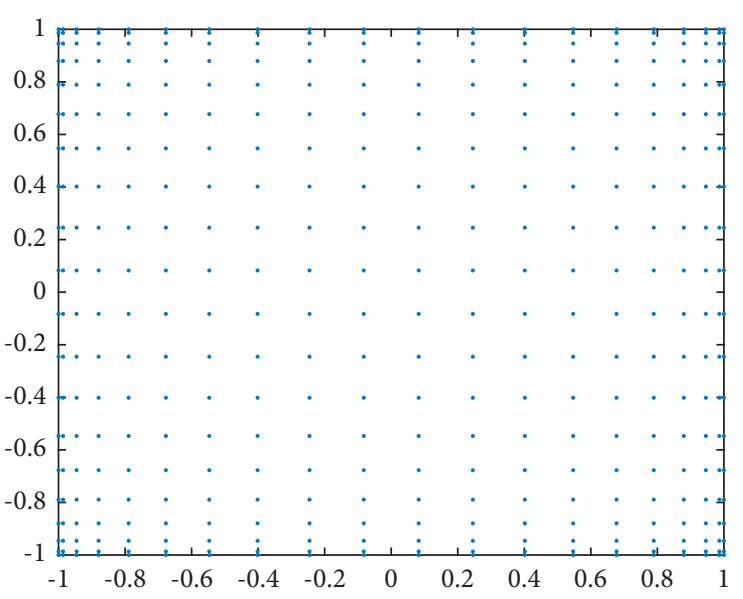

FIgure 1: Configuration of the points in the Chebyshev-type scheme.

By forcing equation (4) to satisfy equations (1)-(3) at all collocation points which are the same as source points $\left\{P_{j}\right\}_{j=1}^{M}$, we have a $M \times M$ system $Q \alpha=b$, where $b=\left(f_{1}, \ldots, \quad f_{M}, \bar{u}_{M+1}, \ldots, \bar{u}_{N_{D}}, \bar{q}_{N_{D}+1}, \ldots, \bar{q}_{N}\right)^{T}, \quad \alpha=$ $\left(\alpha_{1}, \alpha_{2}, \ldots, \alpha_{N}\right)^{T}$, and $(\cdot)^{T}$ is the transpose of vector.

\section{Numerical Results}

In this section, three examples are considered to validate the DRBF. For fair comparison with the other numerical methods, we use the maximum absolute error (MAE), absolute error, and root mean square error (RMSE). The RMSE is defined as follows $[17,18]$ :

$$
\mathrm{RMSE}=\sqrt{\frac{1}{N_{t}-1} \sum_{j=1}^{N_{t}}\left|u\left(x_{j}, t_{j}\right)-\bar{u}\left(x_{j}, t_{j}\right)\right|^{2}},
$$

where $u\left(x_{j}, t_{j}\right)$ is the analytical solution at test points $\left(x_{j}, t_{j}\right), j=1,2, \ldots, N_{t}$, and $\bar{u}\left(x_{j}, t_{j}\right)$ is the numerical solutions at the test points $\left(x_{j}, t_{j}\right), j=1,2, \ldots, N_{t} . N_{t}$ is the number of test points on the physical domain.

3.1. Example 1. We consider the same example as stated in [9]; the exact solution of equations (1)-(3) is

$$
u(x, y)=\sin \frac{\pi x}{6} \sin \frac{7 \pi x}{4} \sin \frac{3 \pi y}{4} \sin \frac{5 \pi y}{4},
$$

and the domain $\Omega=[1,2] \times[1,2]$, and the coefficients of equations (1)-(3) are $a(x, y)=1, b(x, y)=1, c(x, y)=0$, $d(x, y)=0, e(x, y)=0$, and

$$
\begin{aligned}
f(x, y)= & L u(x, y)=-\frac{751 \pi^{2}}{144} \sin \frac{\pi x}{6} \sin \frac{7 \pi x}{4} \sin \frac{3 \pi y}{4} \sin \frac{5 \pi y}{4} \\
& +\frac{7 \pi^{2}}{12} \cos \frac{\pi x}{6} \cos \frac{7 \pi x}{4} \sin \frac{3 \pi y}{4} \sin \frac{5 \pi y}{4}+\frac{15 \pi^{2}}{8} \sin \frac{\pi x}{6} \sin \frac{7 \pi x}{4} \cos \frac{3 \pi y}{4} \cos \frac{5 \pi y}{4} .
\end{aligned}
$$


Here, we abbreviate the MQ with different conical splines as CMQ5 for $r^{5}$, CMQ7 for $r^{7}$, CMQ9 for $r^{9}$, and CMQ11 for $r^{11}$. The collocation point number is chosen under $M_{I}=19 \times 19$ and $M_{b}=M_{D}+M_{N}=80$. The CMQ with Chebyshev-type collocation points is short for CMQC. Since the collocation under the Chebyshev-type is not uniformly distributed, the collocation point number is chosen under $M_{I}=361$ and $M_{b}=M_{D}+M_{N}=76$. As is known to all, the solution accuracy at corner points usually oscillate more serious than the inner points. The calculation point number $N_{t}=$ 40401 is chosen evenly distributed in $[1,2]^{2}$ rather than $[1.1,1.9]^{2}$ used in [9].

Figure 2 shows the RMSEs of numerical results under different values of $c$. As can be seen from Figure 2, the influence of shape parameter is disappeared for $c \geq 1$. Moreover, the influence of collocation numbers on numerical results is also investigated as depicted in Figure 3, where $M=N^{2}+4 N$ for CMQ, $M=N^{2}+4 N-4$ for $\mathrm{CMQC}$, and $c=1$. From Figures 2 and 3, we find that the CMQ9 and CMQC9 perform more stable than the other cases for both shape parameter and collocation numbers, while the CMQC9 case performs the best solution accuracy.

For fixed collocation point number $(M=441$ for CMQ and $M=437$ for CMQC), Figure 4 provides the absolute error surfaces for field solution on the whole solution domain. From which we find that the absolute errors for corner points oscillate for both CMQ5 and CMQ9, but the CMQC9 case can alleviate this phenomenon.

For further investigations, we suggest using the quasioptimal conical spline $r^{9}$ accompanied with collocation points under the Chebyshev-type scheme. This can improve solution accuracy of the CRBF as well as stability with no additional computational cost.

3.2. Example 2. We consider a heat transfer problem with the temperature distribution governed by the following equation:

$$
\nabla \cdot(D \nabla u)+f=0,
$$

and on the same domain as in Example 1 section, the conductivity matrix of the material $D$ is in the following expression:

$$
D=\left(\begin{array}{cc}
(2 x+y+2)^{2} & (x-y)^{2} \\
(x-y)^{2} & (x+2 y+2)^{2}
\end{array}\right) .
$$

More specifically,

$$
\begin{aligned}
\nabla \cdot(D \nabla u)= & (2 x+y+2)^{2} u_{x x}+(x+2 y+2)^{2} u_{y y} \\
& +2(x-y)^{2} u_{x y}+(10 x+2 y+8) u_{x} \\
& +(2 x+10 y+8) u_{y} .
\end{aligned}
$$

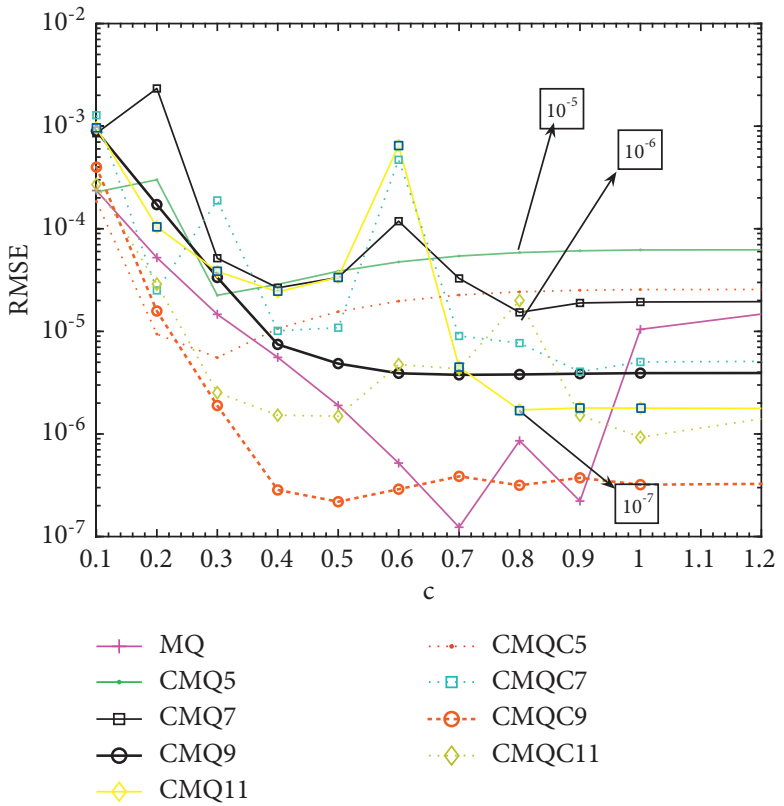

FIGURE 2: RMSEs for field solution versus the value of parameter $c$.

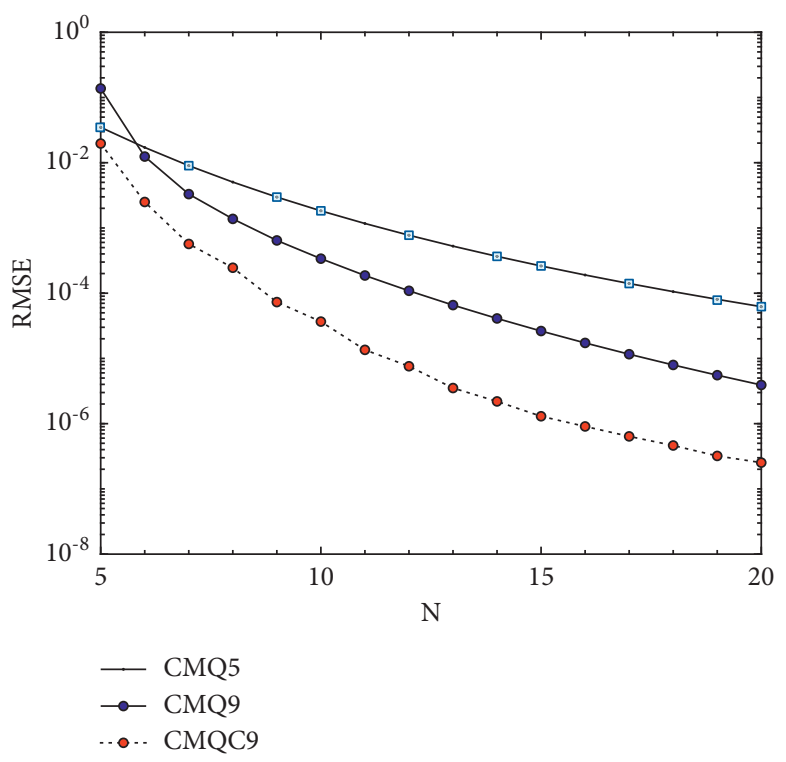

FIgURE 3: RMSEs for field solution versus the collocation number $M$.

The boundary condition is given according to the analytical solution $u=x^{2}+y^{2}-5 x y$, and the corresponding source function is $f=18\left(x^{2}+y^{2}\right)-16$.

Figure 5 shows the RMSEs of numerical results under different values of $c$. As can be seen from Figure 5, the influence of shape parameter is disappeared for $c \geq 0.25$. At the same time, we find that the original MQ method owns more accurate results than the other CMQ methods. 
CMQ5

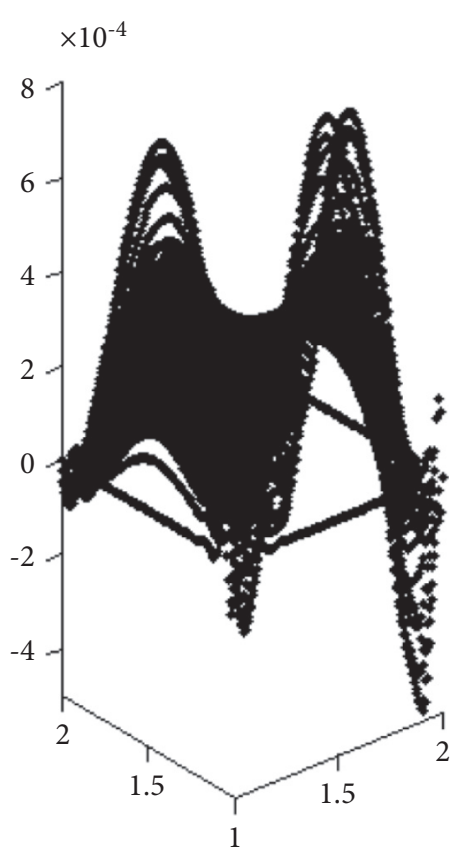

CMQ9

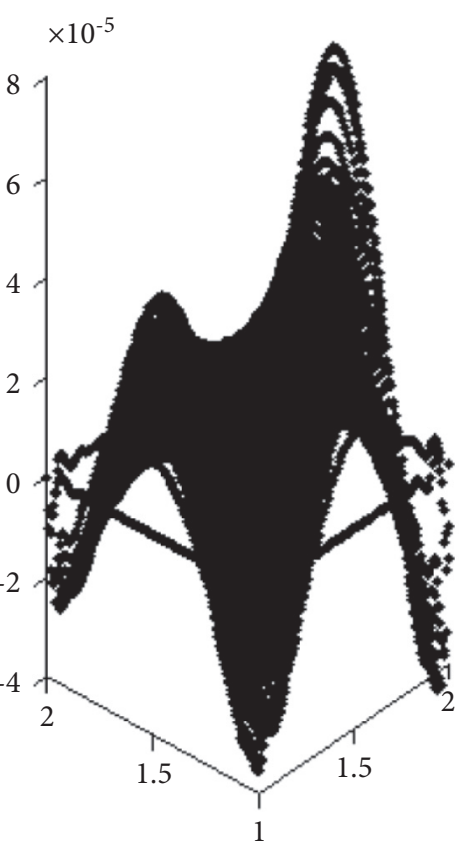

CMQC9

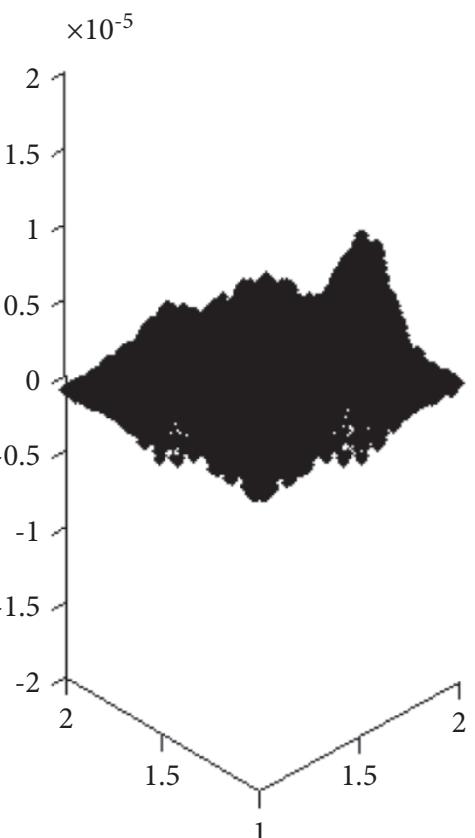

Figure 4: Absolute error surface for field solution on the whole solution domain.

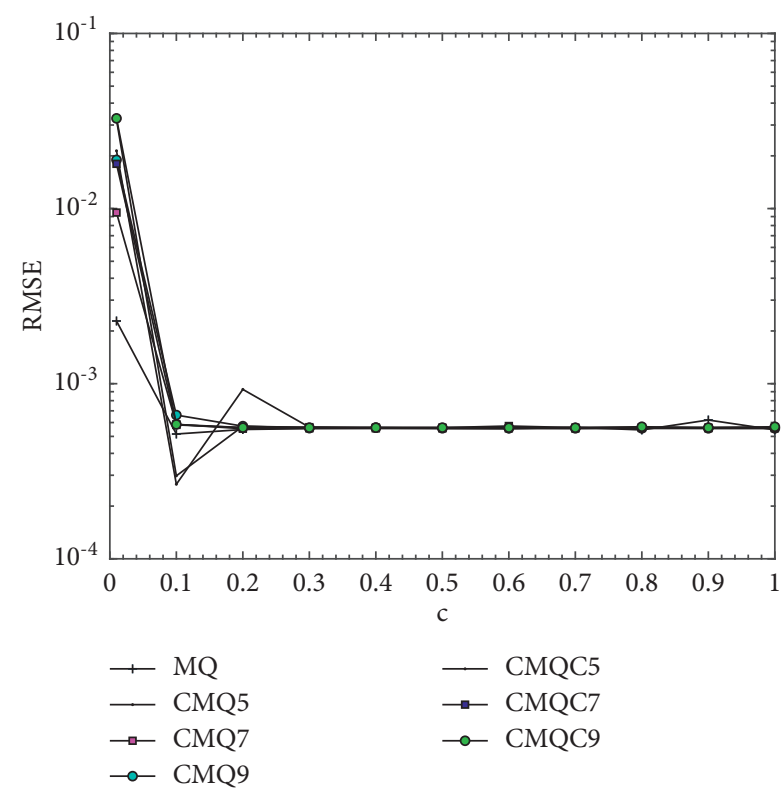

Figure 5: RMSEs for field solution versus the value of parameter $c$.

\section{Conclusions}

The coupled radial basis function, which is based on the infinitely smooth radial basis functions and the conical spline, achieves an accurate and stable numerical solution, while the shape parameter values are almost independent. In this paper, a quasi-optimal conical spline radial basis function, which can improve the numerical results, is investigated. The collocation points in the Chebyshev-type scheme are considered in the whole solution process; this can improve the solution accuracy of the quasi-optimal conical spline radial basis function method with no additional computational cost.

As illustrated in the two examples, the quasi-optimal conical spline radial basis function method maintains almost invariable accuracy and stability as the shape parameter ranges in a wide scope. This is promising in dealing with many practical problems in engineering applications including inverse problems [19]. As a global collocation scheme, the dense matrix is another issue for the large-scale problems. The localized radial basis function method or the other methods will be considered in the future for further investigations [20-22].

\section{Data Availability}

The data used to support the findings of this study are available from the corresponding author upon request.

\section{Conflicts of Interest}

The authors declare that they have no conflicts of interest to report regarding the present study.

\section{Acknowledgments}

This work was supported by the Natural Science Foundation of Anhui Province (Project no. 1908085QA09), the Natural Science Foundation of Shandong Province (Project no. ZR2020ME194), the Open Fund of SMART (No. 201812029), and the University Natural Science Research Project of Anhui Province (Project nos. KJ2018A0396 and KJ2020ZD008). 


\section{References}

[1] E. J. Kansa, "Multiquadrics-a scattered data approximation scheme with applications to computational fluid-dynamics-I surface approximations and partial derivative estimates," Computers \& Mathematics with Applications, vol. 19, no. 8-9, pp. 127-145, 1990.

[2] M. A. Golberg, C. S. Chen, and S. R. Karur, "Improved multiquadric approximation for partial differential equations," Engineering Analysis with Boundary Elements, vol. 18, no. 1, pp. 9-17, 1996.

[3] B. Fornberg and G. Wright, "Stable computation of multiquadric interpolants for all values of the shape parameter," Computers \& Mathematics with Applications, vol. 48, no. 5-6, pp. 853-867, 2004.

[4] F. Z. Wang and K. H. Zheng, "The method of fundamental solutions for steady-state groundwater flow problems," Journal of the Chinese Institute of Engineers, vol. 39, no. 2, pp. 236-242, 2016.

[5] G. B. Wright and B. Fornberg, "Stable computations with flat radial basis functions using vector-valued rational approximations," Journal of Computational Physics, vol. 331, pp. 137-156, 2017.

[6] F. Wang, W. Chen, and L. Ling, "Combinations of the method of fundamental solutions for general inverse source identification problems," Applied Mathematics and Computation, vol. 219, no. 3, pp. 1173-1182, 2012.

[7] J. Zhang, F. Z. Wang, and E. R. Hou, "The conical radial basis function for partial differential equations," Journal of Mathematics, vol. 2020, Article ID 6664071, 7 pages, 2020.

[8] S. Rippa, "An algorithm for selecting a good value for the parameter $c$ in the radial basis function interpolation," $A d$ vances in Computational Mathematics, vol. 11, no. 2, pp. 193-210, 1999.

[9] A. H.-D. Cheng, "Multiquadric and its shape parameter-a numerical investigation of error estimate, condition number, and round-off error by arbitrary precision computation," Engineering Analysis with Boundary Elements, vol. 36, no. 2, pp. 220-239, 2012.

[10] W. Chen, Y. Hong, and J. Lin, "The sample solution approach for determination of the optimal shape parameter in the multiquadric function of the kansa method," Computers \& Mathematics with Applications, vol. 75, no. 8, pp. 2942-2954, 2018.

[11] C.-S. Liu and D. Liu, "Optimal shape parameter in the MQRBF by minimizing an energy gap functional," Applied Mathematics Letters, vol. 86, pp. 157-165, 2018.

[12] Y. Zhang, "An accurate and stable RBF method for solving partial differential equations," Applied Mathematics Letters, vol. 97, pp. 93-98, 2019.

[13] F. Dou, Z.-C. Li, C. S. Chen, and Z. Tian, "Analysis on the method of fundamental solutions for biharmonic equations," Applied Mathematics and Computation, vol. 339, pp. 346-366, 2018.

[14] C. S. Chen, A. Karageorghis, and F. Dou, "A novel RBF collocation method using fictitious centres," Applied Mathematics Letters, vol. 101, Article ID 106069, 2020.

[15] M. Koushki, E. Jabbari, and M. Ahmadinia, "Evaluating RBF methods for solving PDEs using padua points distribution," Alexandria Engineering Journal, vol. 59, no. 5, pp. 2999-3018, 2020.

[16] A. Karageorghis, D. Tappoura, and C. S. Chen, "The kansa RBF method with auxiliary boundary centres for fourth order boundary value problems," Mathematics and Computers in Simulation, vol. 181, pp. 581-597, 2021.

[17] F. Wang, W. Chen, and X. Jiang, "Investigation of regularized techniques for boundary knot method," International Journal for Numerical Methods in Biomedical Engineering, vol. 26, no. 12, pp. 1868-1877, 2010.

[18] I. Ahmad, H. Ahmad, P. Thounthong, Y.-M. Chu, and C. Cesarano, "Solution of multi-term time-fractional PDE models arising in mathematical biology and physics by local meshless method," Symmetry, vol. 12, no. 7, p. 1195, 2020.

[19] F. Wang and Z. Ma, "Meshless collocation method for inverse source identification problems," Advances in Applied Mathematics and Mechanics, vol. 7, no. 4, pp. 496-509, 2015.

[20] H. Zheng, Z. Yang, C. Zhang, and M. Tyrer, "A local radial basis function collocation method for band structure computation of phononic crystals with scatterers of arbitrary geometry," Applied Mathematical Modelling, vol. 60, pp. 447-459, 2018.

[21] H. Zheng, C. Zhou, D. J. Yan, Y. S. Wang, and C. Zhang, "A meshless collocation method for band structure simulation of nanoscale phononic crystals based on nonlocal elasticity theory," Journal of Computational Physics, vol. 408, Article ID 109268, 2020.

[22] I. Ahmad, H. Ahmad, M. Inc, S. W. Yao, and B. Almohsen, "Application of local meshless method for the solution of two term time fractional-order multi-dimensional PDE arsing in heat and mass transfer," Thermal Science, vol. 24, no. Suppl. 1, pp. S95-S105, 2020 